\title{
Crystallographically amorphous ferrimagnetic alloys: Comparing a localized atomistic spin model with experiments
}

\author{
Thomas A. Ostler, ${ }^{*}$ Richard F. L. Evans, and Roy W. Chantrell \\ Department of Physics, University of York, Heslington, York YO10 5DD, United Kingdom \\ Unai Atxitia and Oksana Chubykalo-Fesenko \\ Instituto de Ciencia de Materiales de Madrid, CSIC, Sor Juana Ines de la Cruz, 3, E-28049 Madrid, Spain \\ Ilie Radu ${ }^{\dagger}$ \\ Radboud University Nijmegen, Institute for Molecules and Materials, Heyendaalseweg 135, NL-6525 AJ Nijmegen, The Netherlands and \\ Helmholtz-Zentrum Berlin für Materialien und Energie, BESSY II, Albert-Einstein-Strasse 15, D-12489 Berlin, Germany \\ Radu Abrudan \\ Experimentalphysik IV, Ruhr-Universität Bochum, D-44780 Bochum, Germany \\ Florin Radu \\ Helmholtz-Zentrum Berlin für Materialien und Energie, BESSY II, Albert-Einstein-Strasse 15, D-12489 Berlin, Germany \\ Arata Tsukamoto and A. Itoh \\ College of Science and Technology, Nihon University, 7-24-1 Funabashi, Chiba, Japan \\ Andrei Kirilyuk, Theo Rasing, and Alexey Kimel \\ Radboud University Nijmegen, Institute for Molecules and Materials, Heyendaalseweg 135, NL-6525 AJ Nijmegen, The Netherlands
}

(Received 11 November 2010; revised manuscript received 7 March 2011; published 6 July 2011)

\begin{abstract}
We present a computational model of crystallographically amorphous ferrimagnetic alloys using a stochastic Landau-Lifshitz-Gilbert equation of motion for atomistic spins and an atomistic spin Hamiltonian with Heisenberg exchange. The spontaneous equilibrium magnetization is calculated and a comparison with a mean field model is made. The simulations show excellent agreement with experiments on GdFeCo using x-ray magnetic circular dichroism to determine the individual sublattice magnetizations. The calculated temperature dependence of the magnetization shows a polarization of the Gd sublattice leading to a common Curie temperature, in agreement with the experimental data. The intersublattice exchange is shown to be an important energy transfer channel for ultrafast dynamics.
\end{abstract}

DOI: 10.1103/PhysRevB.84.024407

PACS number(s): 75.50.Gg, 75.78.Jp, 75.10.Hk

\section{INTRODUCTION}

Over the past few decades the demand for fast, reliable, and cheap large-scale data storage has increased drastically. Current trends are displaying a shift toward portable devices, which require both low power and excellent performance. As such, research into these technologies is of great importance. Magnetic storage technologies, such as tape and hard disk drives, currently provide the vast majority of digital storage due to superior price and capacity as compared with competing technologies, such as flash. A great deal of research has been dedicated to heat-assisted magnetic recording ${ }^{1}$ (HAMR), which still faces significant challenges. To improve magnetic storage devices in the future a greater understanding of the intrinsic mechanisms by which magnetization processes occur is required. An important component of the research stimulated by these requirements is a study of the fundamental limits of the switching speed of magnetic materials. The fastest precessional reversal demonstrated experimentally using an external magnetic field ${ }^{2,3}$ is $\sim 100 \mathrm{ps,}$ with similar reversal times achieved using a spin-polarized current. $^{4-6}$
Recent experimental work in the field of magneto-optics has demonstrated that carefully shaped laser pulses can be used to manipulate the magnetization dynamics on the subpicosecond time scale ${ }^{7-12}$ in many materials, including cobalt, iron, nickel, and $\mathrm{GdFeCo}$. However, controllable magnetization switching has only been observed in $\mathrm{GdFeCo}$, and this has stimulated a great deal of effort to attempt on many levels to explain the process. ${ }^{13}$ Recently, Vahaplar et al. ${ }^{13}$ demonstrated that the switching involves a unique "linear" reversal mechanism proposed by Kazantseva et al. ${ }^{14}$ in which the magnetization is reduced to zero followed by a remagnetization into the direction of the optically induced effective magnetic field. This is an especially fast mechanism since it is governed by the longitudinal relaxation time, which can be two orders of magnitude faster than the transverse relaxation time governing normal precessional switching.

It is well known that transition-metal (TM) rare-earth (RE) ferrimagnets used in magneto-optical recording may contain a magnetization compensation point $\left(T_{\mathrm{M}}\right),{ }^{15,16}$ which can be well explained in terms of a two-sublattice model. ${ }^{15,17}$ The static properties can be well described using different models, such as a fixed lattice mixed spin Ising model, ${ }^{18}$ Green's 
function studies, ${ }^{19}$ and also using a mean-field approach. ${ }^{15,17}$ During the 1980's a lot of work was carried out on amorphous RE-TM alloys for magneto-optical recording, ${ }^{20}$ hailed as a storage revolution. The excitement for these materials has recovered in the past few years with the observation of ultrafast magnetization switching using femtosecond laser pulses. ${ }^{10}$ The atomistic model is currently the only model that can describe ultrafast magnetic processes associated with these types of ferrimagnets, however, to the best of our knowledge, the atomistic model has not yet been applied to such disordered ferrimagnets.

In this paper we present an atomistic classical Heisenberg model for a crystallographically amorphous ferrimagnetic alloy. The modeling is based on the Langevin dynamics simulations of the Landau-Lifshitz-Gilbert (LLG) equations for localized atomistic spins. This method of modeling gives us atomic resolution combined with short time-scale dynamical insights into the processes occurring.

In order to construct and validate the model, we compare the results for the static properties of a GdFe-ferrimagnetic alloy of varying composition with $\mathrm{x}$-ray magnetic circular dichroism (XMCD) measurements. In this paper we first present experiments on $\mathrm{GdFeCo}$ which demonstrate the polarization of the $\mathrm{Gd}$ sublattice, leading to a common Curie temperature for the RE and TM spins, agreeing well with the model calculations presented in later sections. It is shown that the Langevin dynamic form of the LLG equation can predict $T_{\mathrm{M}}$ and its effect on the coercivity, which diverges at $T_{\mathrm{M}}{ }^{21,22} \mathrm{It}$ is found that $T_{\mathrm{c}}$ can be adjusted by increasing the exchange between the TM and RE sublattices, and that this also leads to a polarization effect of one sublattice on the other. The model shows qualitative agreement with experiment for temperature-dependent coercivities for a range of TM-RE compositions. A mean-field model is also presented, with a direct comparison being made with the atomistic model. An analytic expression for the Curie temperature $\left(T_{\mathrm{c}}\right)$ is derived and we show the compositional dependence of the $T_{\mathrm{c}}$ and $T_{\mathrm{M}}$. A comparison is made between mean-field results and the LLG model.

We also show that the intersublattice exchange parameter can lead to an effective heating channel, allowing energy to be more rapidly transferred between the two sublattices, shown by calculating the effective spin temperature from equilibrium properties for a range of exchange parameters. This intersublattice exchange might be an important energy transfer mechanism for the ultrafast dynamics observed in these systems, allowing for a linear decrease in the magnetization important when considering such systems at high temperatures.

\section{EXPERIMENTAL DETAILS AND RESULTS}

The element-specific XMCD technique has been employed in order to measure the magnetization of the $\mathrm{Fe}$ and $\mathrm{Gd}$ sublattices in the composite alloy. The XMCD measurements have been performed at the PM3 and UE56/1 beamlines at the synchrotron light source BESSY II Berlin. Both beamlines provide soft $\mathrm{x}$ rays with variable light polarization. XMCD has been measured in transmission geometry with the $\mathrm{x}$ ray at normal incidence and collinear with the external magnetic
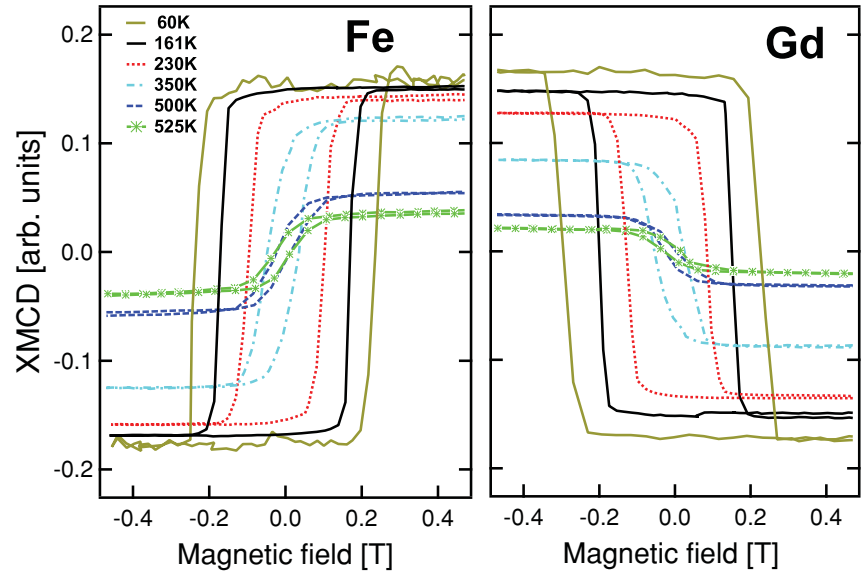

FIG. 1. (Color online) Element- and temperature-dependent hysteresis loops measured for the $\mathrm{Gd}$ and $\mathrm{Fe}$ spins in $\mathrm{Gd}_{23.4} \mathrm{Fe}_{73.3} \mathrm{Co}_{3.3}$ sample using XMCD, clearly showing the antiferromagnetic coupling between the sublattices.

field. The x-ray intensity has been recorded at fixed light helicity for opposite magnetic-field orientations (up to $\pm 0.7 \mathrm{~T}$ ). The difference of the resulting absorption spectra defines the $\mathrm{XMCD}$ value.

The studied samples are amorphous GdFeCo thin films of various stoichiometries grown by magnetron sputtering on a $\mathrm{SiN}$ membrane due to its transparency in the soft x-ray region. A typical sample consists of a 30-nm GdFeCo thin film sandwiched between two layers of SiN (to avoid oxidation), deposited on top of a heat-sink layer of AlTi. The whole structure is deposited on a SiN membrane (150 nm thick) supported by a Si wafer.

Element-specific hysteresis loops have been measured at the $\mathrm{Fe}$ and $\mathrm{Gd}$ absorption edges as a function of temperature to identify the magnetization compensation point, where the coercive field $H_{\mathrm{c}}$ diverges abruptly. The photon energy has been set at the maximum absorption edge ( $L_{3}$ for $\mathrm{Fe}$ and $M_{5}$ for $\mathrm{Gd}$ ) and the transmitted x-ray intensity has been recorded as a function of magnetic field. The resulting temperaturedependent behavior of the hysteresis loops as measured at $\mathrm{Fe}$ and Gd edges is shown in Fig. 1. The opposite polarity of the $\mathrm{Fe}$ and $\mathrm{Gd}$ hysteresis demonstrates the antiferromagnetic alignment of the $\mathrm{Fe}$ and $\mathrm{Gd}$ magnetic moments.

Accounting for the experimental geometry, an out-of-plane magnetization orientation of the sample is deduced from the square shape of the hysteresis, i.e., measurement along the anisotropy easy axis. Moreover, from the measured hysteresis we retrieve the coercivity $H_{\mathrm{c}}$ and the saturation magnetization $M_{\mathrm{S}}$ values at various temperatures. The $M_{\mathrm{s}}$ and $H_{\mathrm{c}}$ measured on a $\mathrm{Gd}_{23.4} \mathrm{Fe}_{73.3} \mathrm{Co}_{3.3}$ sample are displayed in Fig. 2 . The values of the coercivity measured for $\mathrm{Gd}$ and $\mathrm{Fe}$ are similar within the experimental scatter. The variation of $H_{\mathrm{c}}$ with temperature resembles the expected divergent behavior when approaching the compensation point. However, for this particular sample, the compensation point is not crossed within the investigated temperature interval from 60 to $525 \mathrm{~K}$, with just one branch of $H_{\mathrm{c}}(T)$ being measured. At $60 \mathrm{~K}$ the values of $M_{\mathrm{s}}$ for $\mathrm{Fe}$ and $\mathrm{Gd}$ are converging, indicating the close proximity of the magnetization compensation temperature. By fitting the measured $M_{\mathrm{S}}(T)$ data with a power law function $M(T)=M(0)(1-$ 


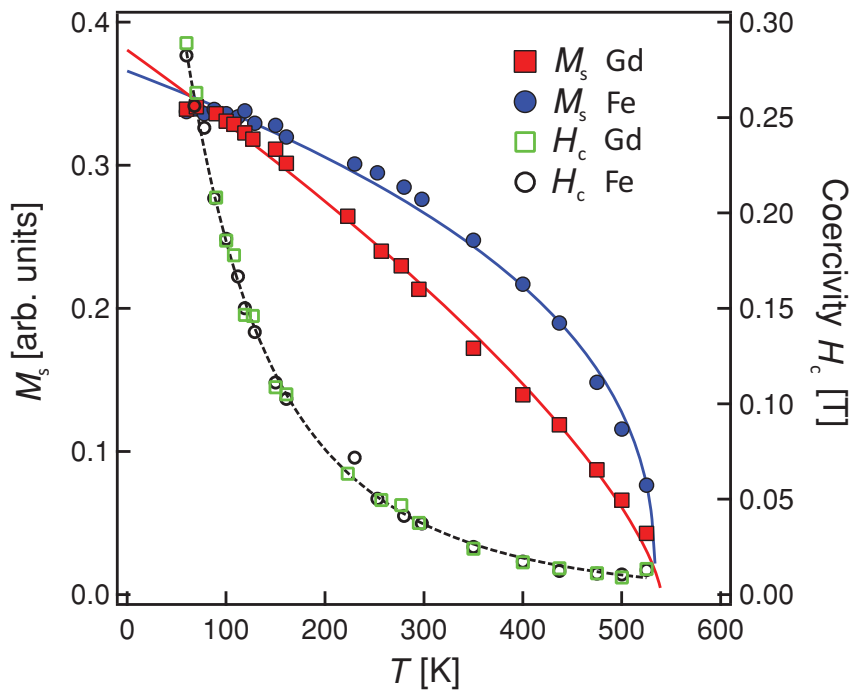

FIG. 2. (Color online) The variation of the coercive field $H_{\mathrm{c}}$ and saturation magnetization $M_{\mathrm{s}}$ with temperature for a $\mathrm{Gd}_{23.4} \mathrm{Fe}_{73.3} \mathrm{Co}_{3.3}$ sample as deduced from element-specific hysteresis measured at the Fe and Gd absorption edges. The divergence in the coercivity indicates the magnetization compensation point. The solid lines are fits according to $M(T)$ power law (see text). Dashed lines are guides to the eye.

$\left.T / T_{\mathrm{c}}\right)^{\rho}$ (the solid lines in Fig. 2) we deduce a common Curie temperature for both $\mathrm{Fe}$ and $\mathrm{Gd}$ sublattices of $540 \pm 10 \mathrm{~K}$. Varying the Gd content from $23.4 \%$ to $29 \%$, we are able to tune the magnetization compensation temperature from below $60 \mathrm{~K}$ to $350 \mathrm{~K}$, as shown in Fig. 3. The experimental results show the coercive field for a range of compositions using the $\mathrm{XMCD}$ technique as described. The divergence of the coercive field represents the magnetization compensation point.

The common Curie temperature for the RE and TM sublattice is an important observation as regards the strength of the exchange coupling between the sublattices. In the following we investigate the effects of intersublattice coupling using the atomistic model.

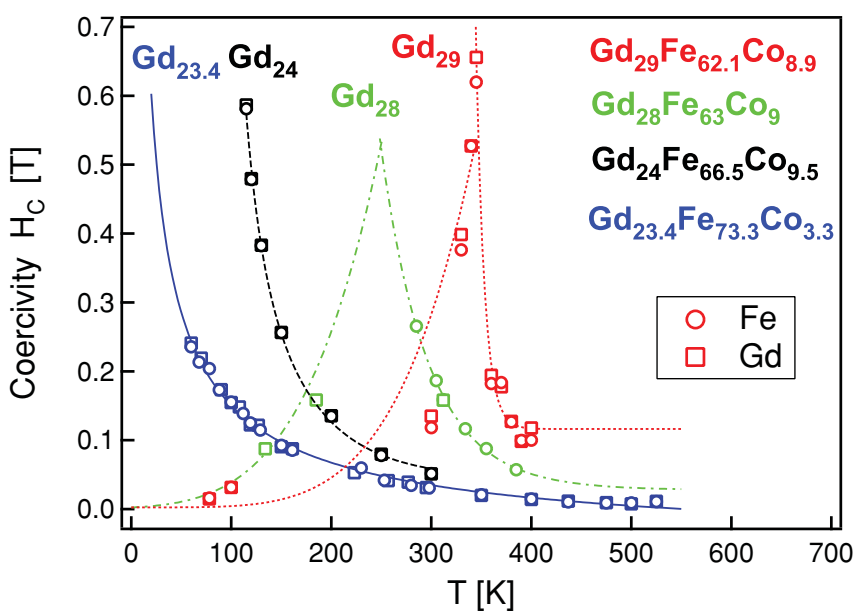

FIG. 3. (Color online) Experimental data showing temperaturedependent coercivity for a range of $\mathrm{GdFeCo}$ compositions. The divergence in the coercivity indicates the magnetization compensation point. The solid lines are guides to the eye.

\section{ATOMISTIC MODEL}

Disordered ferrimagnetic materials demonstrate some very interesting properties, for example, magnetization compensation point, a point at which there is no magnetization below the Curie temperature (for a review, see Ref. 16). Compared with their crystalline counterparts, the amorphous materials can have differing spin moments, a changed band structure, and strikingly different exchange values. In addition, the microscopic origin of the perpendicular anisotropy in $\mathrm{GdFeCo}$ remains elusive and cannot be attributed to strong $L-S$ coupling in Gd as with other RE series in RE-TM hard magnetic materials. Clearly the magnetocrystalline anisotropy is extremely complicated and there have been many suggestions as to its origin including, pair ordering, ${ }^{23}$ single-ion anisotropy, ${ }^{24,25}$ exchange anisotropy ${ }^{26}$ and bond-orientational anisotropy, ${ }^{27}$ with no satisfactory explanation. With this in mind we know that the uniaxial component of the magnetocrystalline anisotropy is dominant in the composition range where the compensation point occurs, therefore in our model we assume a uniaxial anisotropy energy of $8.07246 \times 10^{-24}$ Joules per atom. This value should be strong enough to support perpendicular magnetization in the thin films studied experimentally. For simplicity we choose a generic transition-metal ferromagnet to represent the $\mathrm{Fe}(\mathrm{TM})$, and a separate rareearth ferromagnet for the Gd (RE). The system consists of $N \times N \times N$ fcc cells with periodic boundary conditions. We then populate the fcc lattice with a random distribution of TM and RE ions in the desired concentration $q$ and $x$, respectively $(q+x=1)$. Note here the use of the fcc lattice, this structure of course does not take into account the size of the Gd atom or the fact that the structure is disordered, though the amorphous structure is densely packed and the number of neighbours will not be limited to six. This means that the distance between spins is not realistically taken into account, though this is not important as it does not appear in our Hamiltonian. A more complicated model would require the use of some kind of structural relaxation, though this would be complicated as the exchange parameters would have to be calculated using $a b$ initio techniques requiring averaging over a number of starting configurations.

Using the Heisenberg form of the exchange for nearest neighbors, the energetics of the system are described by the following Hamiltonian:

$$
\mathcal{H}=-\frac{1}{2} \sum_{\langle i, j\rangle} J_{i j} \mathbf{S}_{i} \cdot \mathbf{S}_{j}-\sum_{i=1}^{\mathcal{N}} D_{i}\left(\mathbf{S}_{i} \cdot \mathbf{n}_{i}\right)^{2}-\sum_{i=1}^{\mathcal{N}} \mu_{i} \mathbf{B} \cdot \mathbf{S}_{i},
$$

where $J_{i j}$ is the exchange integral between spins $i$ and $j(i, j$ are lattice sites), $\mathbf{S}_{i}$ is the normalized vector $\left|\mathbf{S}_{i}\right|=1, D_{i}$ is the uniaxial anisotropy constant (assumed along $z$ ), $\mathbf{n}_{i}$ is the direction of the anisotropy vector, $\mu_{i}$ is the magnetic moment of the site $i, \mathbf{B}$ is the vector describing the applied field, and $\mathcal{N}$ is the total number of spins. We model the magnetization dynamics of the system via the use of the LLG equation ${ }^{28}$ with Langevin dynamics, given by

$$
\frac{d \mathbf{S}_{i}}{d t}=-\frac{\gamma_{i}}{\left(1+\lambda_{i}^{2}\right) \mu_{i}}\left(\mathbf{S}_{i} \times \mathbf{H}_{i}^{\mathrm{eff}}+\lambda_{i} \mathbf{S}_{i} \times\left[\mathbf{S}_{i} \times \mathbf{H}_{i}^{\mathrm{eff}}\right]\right) .
$$


Here $\lambda_{i}$ and $\gamma_{i}$ are the Gilbert damping parameter and the gyromagnetic ratio, respectively. The effective field $\mathbf{H}_{i}^{\text {eff }}$ is then given by

$$
\mathbf{H}_{i}^{\mathrm{eff}}=\zeta_{i}(t)-\frac{\partial \mathcal{H}_{i}}{\partial \mathbf{S}_{i}}
$$

Here $\zeta_{i}(t)$ represents a stochastic term, which describes the coupling to the external heat bath. The thermal fluctuations are included as a white-noise term, uncorrelated in time, which is added into the effective field. This form of the noise is treated as a Stratanovich stochastic process. ${ }^{29}$ The thermal fields are calculated by generating Gaussian random numbers and multiplying by the strength of the noise process. The correlators of different components of this field can be written as

$$
\left\langle\zeta_{i, \alpha}(t) \zeta_{j, \beta}\left(t^{\prime}\right)\right\rangle=\frac{2 \lambda_{i} k_{\mathrm{B}} T \mu_{i}}{\gamma_{i}} \delta_{i j} \delta_{\alpha \beta} \delta\left(t-t^{\prime}\right),
$$

where $\alpha, \beta$ are Cartesian components and $T$ is the temperature of the heat bath to which the spin is coupled. The coupling of the spins to the heat bath $(\lambda)$ is a parameter which attempts to describe all of the energy and momentum transfer channels into the spin system, for example, from the lattice and conduction electrons. Note that there is a subtle difference between a local (microscopic) damping parameter $\lambda$ and a macroscopic damping parameter, as measured for a material in an experiment, usually denoted as $\alpha .{ }^{30}$ Although the intrinsic damping is also known to be temperature dependent, ${ }^{31}$ this intrinsic temperature dependence is normally ignored in the modelling of magnetisation dynamics. ${ }^{32,33}$ The intrinsic damping should not be confused with extrinsic which additionally increases with temperature due to the influence of spin disordering. The extrinsic damping is the effective rate for a collection of atomic moments, and depends on temperature, structure, dilution and many other physical parameters, and is unknown a priori. A description of the methods is given in Ref. 33 .

The use of a two sublattice model for modelling GdFeCo is justified by the fact that experimentally we measure a parallel alignment of the $\mathrm{Fe}$ and $\mathrm{Co}$ sublattice up to the Curie temperature. Also the fact that we are using a localised spin model, it would be inappropriate for this type of model, perhaps, to distinguish between the Fe and Co sublattice due to the delocalised nature of their moments. Thus we treat the system as two sublattices, one being FeCo and the other, Gd. Furthermore, the inclusion of a third species would bring about yet more free parameters, in the form of the exchange integrals between the relevant species. We point out at this point that the amount of Co used in the samples in small and thus we can treat the FeCo sublattice as a single species; a generic transition metal.

Because we have two different species, there are some subtleties with regard to the implementation of the model due to the presence of on-site parameters that enter into the LLG equation. Such on-site parameters include $\lambda_{i}, \mu_{i}$, and $\gamma_{i}$, as well as three types of exchange interactions $J_{\mathrm{TM}-\mathrm{TM}}, J_{\mathrm{RE}-\mathrm{RE}}$, and $J_{\mathrm{TM}-\mathrm{RE}}$. Experimentally there is a difference in the effective gyromagnetic ratio of each species due to inhomogeneities in the crystal-field potential. ${ }^{34}$ The effect of different gyromagnetic ratios is the existence of the temperature at which the ratio $M_{1} / \gamma_{1}-M_{2} / \gamma_{2}$ goes to zero, known as the angular momentum compensation temperature $T_{\mathrm{A}}$. At $T_{\mathrm{A}}$ there is no angular momentum associated with the magnetization, which can thus be moved by the slightest torque. ${ }^{34}$ For simplicity, we have assumed that each sublattice has the same gyromagnetic ratio of $1.76 \times 10^{11}(\mathrm{~T} \mathrm{~s})^{-1}$. No data are available for the value of the intrinsic damping of the system, although we hypothesize that the on-site damping constant could be different for the two species. In this model we assume that the damping on each site is equal at $\lambda_{\mathrm{TM}}=\lambda_{\mathrm{RE}}=$ 0.1 . The actual choice of the parameter $\lambda$ is not important for calculation of the static processes. The magnetic moments are of course different; here we use the value for bulk Fe $\left[\mu_{\mathrm{TM}}=2.217 \mu_{\mathrm{B}}\right.$ (Ref. 35) $]$ for the $\mathrm{TM}$ sublattice, where $\mu_{\mathrm{B}}$ is the Bohr magneton. For the RE sites we also use the bulk value of $\mu_{\mathrm{RE}}=7.63 \mu_{\mathrm{B}} .{ }^{36}$ The actual moments will vary with composition because of, for example, hybridization effects. For instance, from the XMCD measurements we see slightly smaller Fe moments, however, because of the complexity of the value of the moments with each composition, we keep a constant value in the theoretical model.

\section{SIMULATION RESULTS AND MEAN-FIELD MODEL}

In the following we present calculations of the magnetic properties of a TM-RE system using the atomistic model, including temperature-dependent magnetization and coercivities. The results show excellent qualitative agreement with the XMCD experimental data. We note here that the experimental data is for GdFeCo with a mixed TM sublattice, whereas our model has a single RE and is, in principle, valid for GdFe. However, it is reasonable to assume that the FeCo forms a single sublattice, allowing a reasonable comparison between the model predictions and the experiments. XMCD measurements demonstrate a ferromagnetic alignment of the $\mathrm{Fe}$ and $\mathrm{Co}$ sublattices in the GdFeCo alloy up to the Curie temperature.

\section{A. Temperature-dependent magnetization}

As a first approximation we have used bulk exchange values for neighboring TM ions $\left(J_{\mathrm{TM}-\mathrm{TM}}=4.5 \times 10^{-21} \mathrm{~J}\right)$ and for neighboring $\mathrm{RE}$ ions $\left(J_{\mathrm{RE}-\mathrm{RE}}=1.26 \times 10^{-21} \mathrm{~J}\right)$, which give the correct Curie temperature $T_{\mathrm{c}}$ for the separate sublattices. The validity of this approximation of bulk exchange is questionable because of the changing interatomic distances and coordination numbers. However, this allows for a systematic study over a range of compositions. Were one to study a particular composition, this model would allow us to change the exchange values to fit any available $a b$ initio or experimental data. The value of the intersublattice exchange $J_{\mathrm{TM}-\mathrm{RE}}=-1.09 \times 10^{-21} \mathrm{~J}$ was chosen so that the temperature dependence of the TM and RE sublattices agreed qualitatively with results of XMCD measurements of static magnetization, shown in Fig. 2. This will be discussed later in more detail. Due to the larger atomic radius of $\mathrm{Gd}$, it is possible that the TM-TM exchange is reduced because of the fact that as the amount of RE is increased, the distance between the TM ions increases. However, in the absence of any detailed information about the spatial dependence of the exchange, these effects are difficult to quantify. 


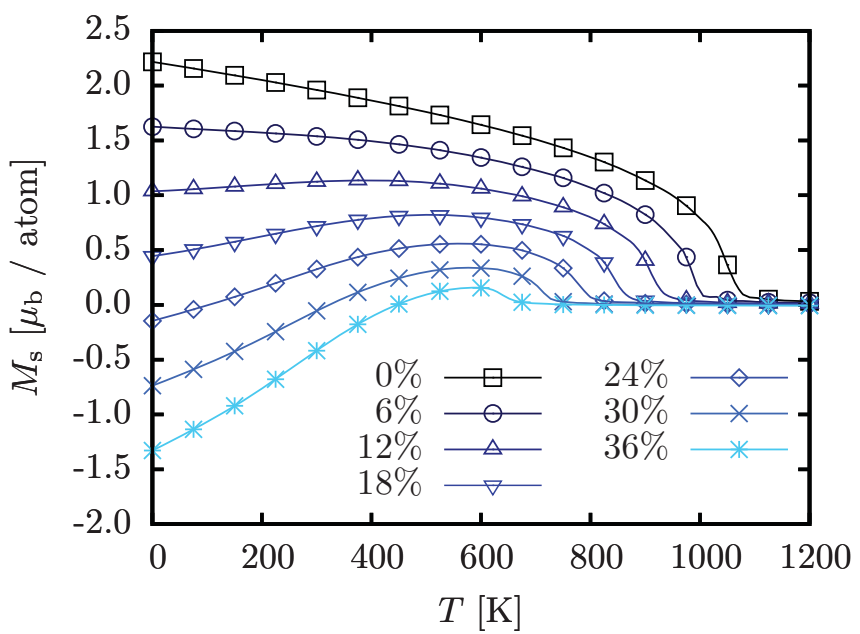

FIG. 4. (Color online) Numerically calculated magnetization curves for the TM-RE ferrimagnet as a function of temperature. Results are shown for a range of RE content. The compensation point exists for concentration in the range $18 \%-44 \%$, consistent with experiment (Ref. 16). Note that the sign of $M_{\mathrm{s}}$ takes into account the fact that the TM sublattice is pointing opposite to the RE sublattice. In the negative region, therefore, the RE sublattice is dominant.

The model is capable of reproducing the key magnetic properties specifically the temperature-dependent magnetization, as shown in Fig. 4. These results were obtained by simulating a system of 62500 spins with periodic boundary conditions. The system is equilibrated until there is only a small change in the magnetization for each temperature point. The thermodynamic average of the magnetization was calculated over a further period of $t_{\max }=60 \mathrm{ps,}$

$$
\bar{m}_{i}=\frac{1}{t_{\max }} \sum_{t=0}^{t_{\max }} m_{i}(t),
$$

where $m_{i}$ is the reduced magnetization of the sublattice $i$. Considering first the data in Fig. 4, at high RE concentrations ( $x$ ) there is a compensation point, i.e., a point below $T_{\mathrm{c}}$ at which the total magnetization crosses zero. This point occurs at a temperature which decreases with decreasing $x$. Thus, the model is behaving in a way consistent with the experiment. ${ }^{16}$ With decreasing $x$, the compensation temperature decreases, vanishing at a critical concentration $x_{c}$. For values of $x<x_{c}$ the magnetization shows a peak value at some temperature greater than zero. This results from the competing exchange coupling terms, with the ferromagnetic exchange becoming important at low $x$. The addition of RE also acts to reduce the Curie temperature.

As mentioned, the compensation point does not exist for compositions greater than 44\%. Figure 5 shows the magnetization curves for $x>44 \%$ up to pure RE, which for $\mathrm{Gd}$, as mentioned, has the correct Curie temperature. The orientation of the sublattices is reversed in this figure with the RE sublattice pointing in the positive direction.

\section{B. Mean-field approximation}

In this section we present an adapted mean-field approximation (MFA) to the free energy of the system, ${ }^{15,21,37,38}$ which we

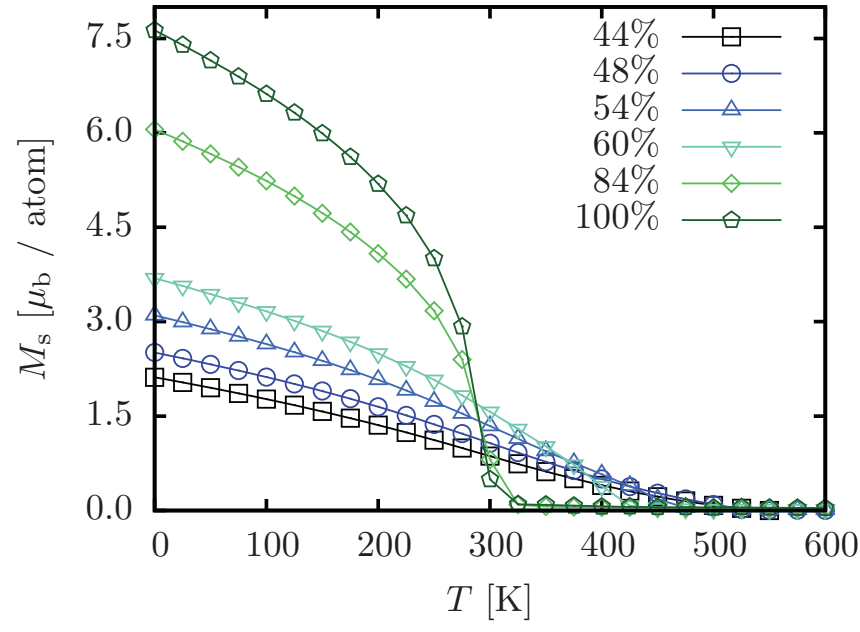

FIG. 5. (Color online) Numerically calculated magnetization curves for the TM-RE ferrimagnet as a function of temperature. Results are shown for a range of RE content. This figure shows that the compensation point does not exist above $44 \%$ RE. Note that the sign of $M_{\mathrm{s}}$ takes into account the fact that the TM sublattice is pointing opposite to the RE sublattice. All of the data are plotted as the absolute value of the magnetization as there does not exist a compensation point in this range.

use to verify the atomistic model and to make a comparison of the Curie temperatures and magnetization compensation point. We extract the equilibrium magnetization of each sublattice and compare the results with those obtained from the numerical model.

The free energy $\mathcal{F}=-k_{\mathrm{B}} T \ln \mathcal{Z}$ of a spin system ( $\mathcal{Z}$ is the partition function) described by the Hamiltonian in Eq. (1) can be calculated in the MFA by considering each spin on a site $i$ as an isolated spin, with the effective field containing contributions determined by the mean values of the neighboring ones. ${ }^{39}$ Here we are dealing with a special case of a two-sublattice model. We can subdivide the lattice into two asymmetrical sublattices, the impurity sublattice with RE spins and the bulk one with TM spins. Namely,

$$
\begin{aligned}
\mathcal{H} \Rightarrow & \mathcal{H}^{\mathrm{MFA}}=\mathcal{H}_{00}-\mu_{\mathrm{TM}} \sum_{\mathrm{TM}} \mathbf{H}_{\mathrm{TM}}^{\mathrm{MFA}} \cdot \mathbf{s}_{\mathrm{TM}} \\
& -\mu_{\mathrm{RE}} \sum_{\mathrm{RE}} \mathbf{H}_{\mathrm{RE}}^{\mathrm{MFA}} \cdot \mathbf{s}_{\mathrm{RE}},
\end{aligned}
$$

where $\mathcal{H}_{00}=\sum_{i j} J_{i j} \boldsymbol{\sigma}_{i} \cdot \boldsymbol{\sigma}_{j}+\sum_{i} D_{i} \sigma_{z i}^{2}$, with $\quad \boldsymbol{\sigma}_{i} \equiv\left\langle\mathbf{s}_{i}\right\rangle$ being the spin polarization of the site $i$, and the molecular field $\mathbf{H}_{i}^{\mathrm{MFA}}$ is given by

$$
\mu_{i} \mathbf{H}_{i}^{\mathrm{MFA}}=\mu_{i} \mathbf{H}+2 D_{i} \sigma_{z i} \mathbf{e}_{z}+\sum_{j} J_{i j} \boldsymbol{\sigma}_{j} .
$$

Now, the exchange part is treated by first dividing the sum over the neighbors in two, i.e., $\sum_{j} \longrightarrow \sum_{\mathrm{TM}}+\sum_{\mathrm{RE}}$. Next we define the anisotropy field as $\mathbf{H}_{A, i}=\left(2 D_{i} / \mu_{i}\right) \sigma_{i, z} \mathbf{e}_{z}$ and pass to the continuous limit $\sigma_{i} \Rightarrow \boldsymbol{\sigma}(\mathbf{r})$, assuming $\Delta \boldsymbol{\sigma}(\mathbf{r})=0$. Then the molecular field reads

$$
\mu_{i} \mathbf{H}_{i}^{\mathrm{MFA}}=\mu_{i}\left(\mathbf{H}+\mathbf{H}_{A, i}\right)+\sum_{\mathrm{TM}} J_{i}^{\mathrm{TM}} \boldsymbol{\sigma}_{\mathrm{TM}}+\sum_{\mathrm{RE}} J_{i}^{\mathrm{RE}} \boldsymbol{\sigma}_{\mathrm{RE}} .
$$


If $z$ is the number of nearest neighbors and $x$ is the RE concentration, then a TM moment will have on average $z x$ neighboring RE moments and $z(1-x)=z q$ neighboring TM moments and conversely for the RE moments. Averaging over the system configurations ${ }^{40}$ and defining $\mathbf{H}_{\text {eff,i }}^{\prime}=\mathbf{H}+\mathbf{H}_{A, i}$, we can write the average molecular field acting at each sublattice spin as

$$
\begin{gathered}
\mu_{\mathrm{RE}} \mathbf{H}_{\mathrm{RE}}^{\mathrm{MFA}}=\mu_{\mathrm{RE}} \mathbf{H}_{\mathrm{eff}, \mathrm{RE}}^{\prime}+x J_{0, \mathrm{RE}-\mathrm{RE}} \boldsymbol{\sigma}_{\mathrm{RE}}+q J_{0, \mathrm{TM}-\mathrm{RE}} \boldsymbol{\sigma}_{\mathrm{TM}}, \\
\begin{aligned}
\mu_{\mathrm{TM}} \mathbf{H}_{\mathrm{TM}}^{\mathrm{MFA}}= & \mu_{\mathrm{TM}} \mathbf{H}_{\mathrm{eff}, \mathrm{TM}}^{\prime}+q J_{0, \mathrm{TM}-\mathrm{TM}} \boldsymbol{\sigma}_{\mathrm{TM}} \\
& +x J_{0, \mathrm{TM}-\mathrm{RE}} \boldsymbol{\sigma}_{\mathrm{RE}},
\end{aligned}
\end{gathered}
$$

where $J_{0, i j}=z J_{i j}$. Note that to recover the Hamiltonian for the pure ferromagnet in Eqs. (6) and (5), we must set $x=0$ and $J_{0, \mathrm{TM}-\mathrm{TM}}=0$. The free energy takes the form

$$
\mathcal{F}=\mathcal{H}_{00}-\mathcal{N} k_{\mathrm{B}} T \ln (4 \pi)-k_{\mathrm{B}} T \sum_{i} \Lambda\left(\xi_{i}\right),
$$

where $\quad \Lambda(\xi) \equiv \ln (\sinh (\xi) / \xi), \quad \xi_{i} \equiv\left|\xi_{i}\right|, \quad$ and $\quad \xi_{i} \equiv$ $\mu_{i} \mathbf{H}_{i}^{\mathrm{MFA}} / k_{\mathrm{B}} T$. The mean-field free energy determined by Eqs. (5)-(7) can be minimized with respect to the spin averages $\sigma_{\mathrm{RE}}$ and $\sigma_{\mathrm{TM}}$ to find the equilibrium solution. The minimum condition for the free energy $\partial \mathcal{F} / \partial \sigma_{\mathrm{RE}}=0$ and $\partial \mathcal{F} / \partial \sigma_{\mathrm{TM}}=0$ leads to the coupled Curie-Weiss equations

$$
\boldsymbol{\sigma}_{\mathrm{RE}}=L\left(\xi_{\mathrm{RE}}\right) \frac{\boldsymbol{\xi}_{\mathrm{RE}}}{\xi_{\mathrm{RE}}}, \quad \boldsymbol{\sigma}_{\mathrm{TM}}=L\left(\xi_{\mathrm{TM}}\right) \frac{\boldsymbol{\xi}_{\mathrm{TM}}}{\xi_{\mathrm{TM}}},
$$

where $L(\xi)=\operatorname{coth}(\xi)-1 / \xi$ is the Langevin function. Then the equilibrium magnetization of each sublattice can be obtained via the self-consistent solution of Eqs. (8). The total equilibrium magnetization per atom is evaluated as

$$
M=\mu_{\mathrm{TM}} q \sigma_{e, \mathrm{TM}}-\mu_{\mathrm{RE}} x \sigma_{e, \mathrm{RE}} .
$$

Close to $T_{\mathrm{c}}$ we may expand $L(\xi) \cong \xi / 3$, and calculate $T_{\mathrm{c}}^{\mathrm{MFA}}$ of the ferrimagnet. We obtain the equation

$$
T_{\mathrm{c}}(x)^{\mathrm{MFA}}=\frac{2 a}{3 k_{\mathrm{B}} b} \frac{1}{\sqrt{1+4 a / b^{2}}-1},
$$

where

$$
\begin{gathered}
a=q x\left(J_{0, \mathrm{TM}-\mathrm{RE}}^{2}-J_{0, \mathrm{RE}-\mathrm{RE}} J_{0, \mathrm{TM}-\mathrm{TM}}\right), \\
b=q J_{0, \mathrm{TM}-\mathrm{TM}}+x J_{0, \mathrm{RE}-\mathrm{RE} .}
\end{gathered}
$$

In the low concentration limit we obtain a linear decrease of the Curie point $T_{\mathrm{c}}^{\mathrm{MFA}}=q J_{0, \mathrm{TM}-\mathrm{TM}} / 3$.

Figure 6 presents a comparison between the MFA and LLG model for the total magnetization as a function of temperature for different TM-RE concentrations. The MFA as always overestimates the value of the Curie temperature. Following the conventional comparison, the data in the atomistic model are normalized to the corresponding Curie temperature for each composition.

To compare the MFA results with the predictions of the atomistic model, Fig. 7 includes the Curie temperature calculated using the Eq. (10) with rescaled interaction $J_{i j}$ to fit the exact values in the pure ferromagnetic (antiferromagnetic) limits, $3.18 \mathrm{~J}=k_{\mathrm{B}} T_{\mathrm{c}}$ for face centred cubic structures. ${ }^{41}$ In the LLG model, to calculate the Curie temperature in a consistent way, to overcome the problem of finite-size effects,

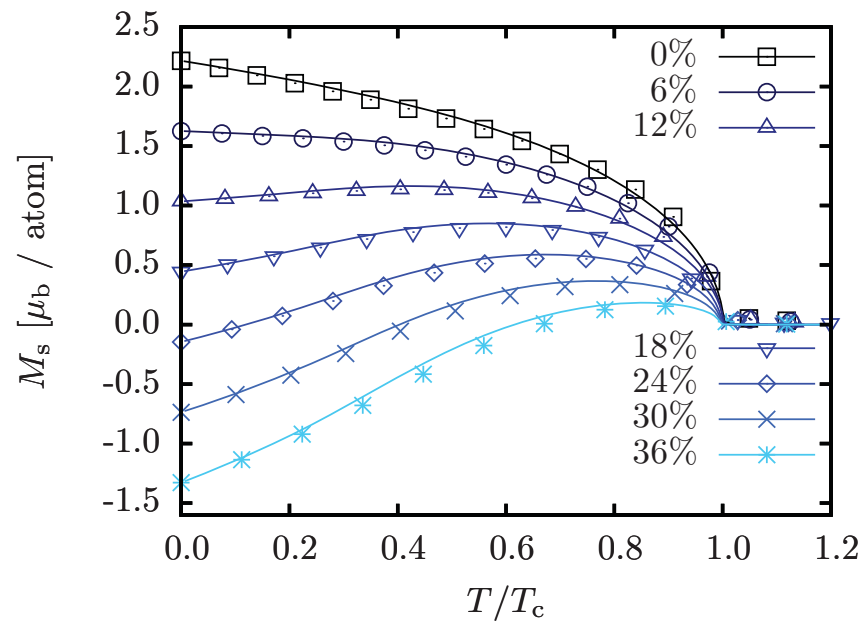

FIG. 6. (Color online) Magnetization curves as a function of temperature (normalized to the Curie temperature $T_{\mathrm{c}}$ for each composition) for different concentrations of RE. The data is calculated via the MFA approach (solid lines) and direct integration of the Langevin dynamics equation (2) (symbols).

we performed a fit to the numerical data using the same method as in Ref. 42, whereby the data were interpolated to $M=0$ - this point represents the phase transition. We have also compared the calculated compensation temperature (where it exists) as a function of composition. The results are shown in Fig. 7.

As can be seen in Fig. 7, the LLG model agrees very well with the MFA predictions. The magnetization compensation point appears at the same composition in both models and disappears again at around the same point, showing the strength of the modified mean-field model for predicting static properties. The composition range for which the magnetization

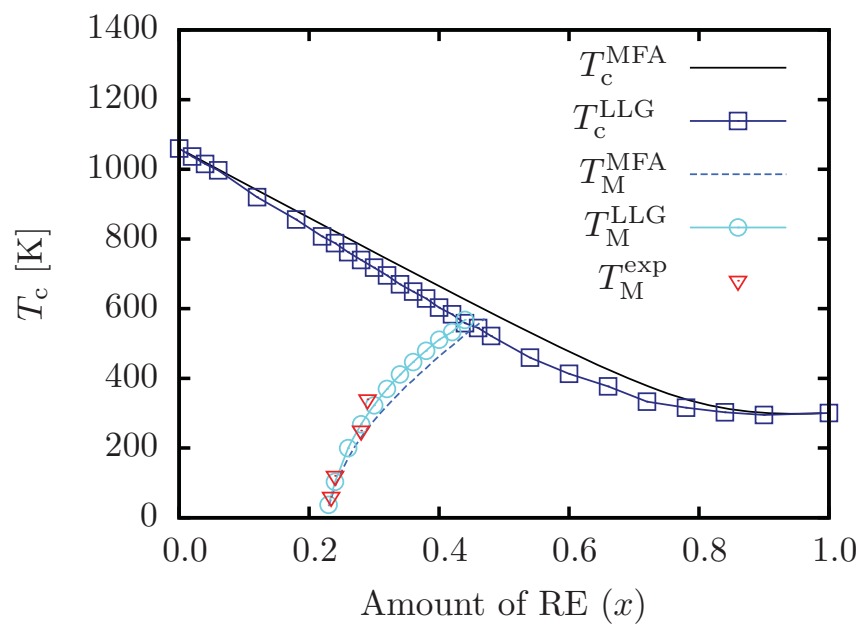

FIG. 7. (Color online) Compositional dependence of the Curie temperature $\left(T_{\mathrm{c}}\right)$ and magnetization compensation point $\left(T_{\mathrm{M}}\right)$. The mean-field approximation (MFA) with renormalized exchange parameters is shown to agree very well (lines) with the LandauLifshitz-Gilbert (LLG) model (points). The compensation temperatures deduced from temperature-dependent hysteresis curves show excellent agreement with the mean field and LLG models. 


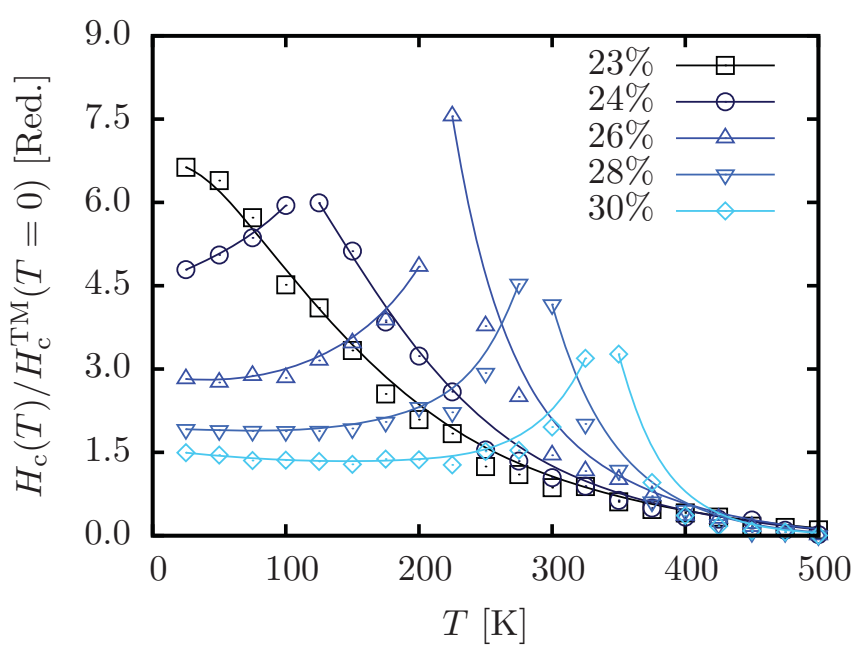

FIG. 8. (Color online) Numerical values of the coercive field (points) for various RE amounts. Data shown was calculated using a sweep rate of $1 \mathrm{~T} / \mathrm{ns}$ for a range of compositions. Results show good qualitative agreement with the experimental results (Fig. 3), with the divergence representing the magnetization compensation point. Lines are guides to the eye. Values are reduced to the zero-temperature, pure TM coercivity value with the same sweep rate.

compensation point exists is also in good agreement with the experimental data in Ref. 16.

These initial results act as a validation of the computational model and mean-field approach. Next the atomistic model is used in a study of the effects of interlayer coupling on the static and dynamic properties of the model ferrimagnet.

\section{Coercivity calculations}

Using the LLG model we show the compositional dependence on the coercivity. The model reproduces qualitatively similar behavior to the experimental results shown in Fig. 3. The systems modeled are 62500 spins in size due to limits on computational resources, therefore, a single domain state exists and reversal occurs via precessional switching over the energy barrier. Figure 8 shows the results of numerical calculations of the coercive field for a range of compositions of the TM-RE system. The sweep rate applied was $1 \mathrm{~T} / \mathrm{ns}$, which was required for computational efficiency. The system was first equilibrated at the given temperature and then the field was ramped in the opposing direction to the dominant sublattice. The lines are guides to the eye applied above and below $T_{\mathrm{M}}$ for each composition. Qualitative agreement with the experimental results of Fig. 3 is good, showing that the divergence is due to the magnetization compensation point. This is another validation of the use of this simple atomistic model as a first approximation for this type of Ferrimagnet. Complete agreement between experiment and theory is not possible at the moment as the coercivity of a material depends on many things, amongst other things, the presence of defects, morphology, chemical segregation, formation of magnetic grains, interfacial properties and the time over which the field is swept, i.e. it is a time dependent quantity. Quantitative agreement between the LLG simulations and experiments for the whole range of temperatures and compositions is therefore highly computationally expensive. This is because of the

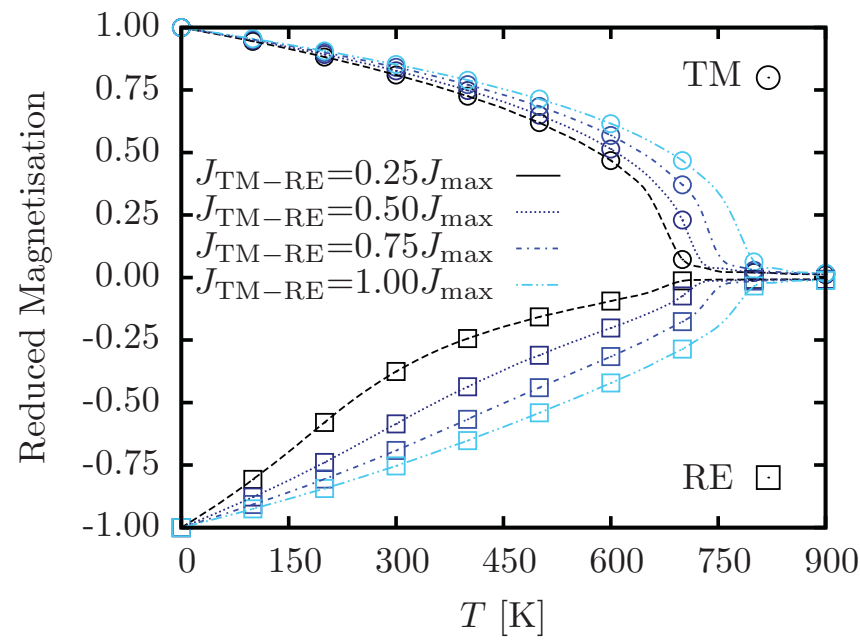

FIG. 9. (Color online) Reduced magnetization of the Fe and Gd sublattices as a function of the intersublattice exchange $\left(J_{\mathrm{TM}-\mathrm{RE}}\right)$. Magnetization is normalized to the sublattice magnetization. The exchange leads to a polarization effect between the sublattices. Here $J_{\max }=-2.18 \times 10^{-21} \mathrm{~J}$.

timescales involved in the experiments, which are currently unreachable by the LLG model. With this in mind we still see the effect on the coercivity of the magnetization compensation point in both the experiments and the simulations with this very simple model

\section{Computational results for the effect of TM-RE exchange}

As previously discussed, the temperature dependence of the magnetization of each sublattice is different depending on the effective exchange. Although the details of the exchange parameters are unknown, we can get an insight into the strength of the exchange between the sublattices by comparing the results of our simulations with the XMCD experiments described earlier, which measure the magnetization of each sublattice.

It can be seen from Fig. 2 that the magnetization is almost linear with temperature for the RE sublattice, and the two sublattices show the same Curie temperature $T_{\mathrm{c}}$. To get an insight into the strength of the exchange between the two sublattices, we employed the Langevin dynamics model of the TM-RE ferrimagnet to calculate the reduced static magnetization as a function of the intersublattice exchange parameter $J_{\mathrm{TM}-\mathrm{RE}}$. Other than the variable intersublattice exchange, the simulation details are the same as for the results in Fig. 4. The results are shown in Fig. 9, which shows the reduced magnetization of the Fe and Gd sublattices as a function of the intersublattice exchange coupling $\left(J_{\mathrm{TM}-\mathrm{RE}}\right)$. Over the range of exchange coupling shown, in agreement with Fig. 2, the two sublattices share the same Curie temperature, suggesting that there is a polarization effect of one sublattice on the other. This polarization effect also changes the temperature dependence of the magnetization, as seen in Fig. 9. For weaker coupling (not shown here), the RE sublattice shows a reduced $T_{\mathrm{c}}$. The experimental results in Fig. 2 clearly show the presence of strong coupling between the sublattices. Comparison between the calculations and experimental results suggest a value of $\sim-1.09 \times 10^{-21} \mathrm{~J}$. This factor is potentially important in relation to ultrafast magnetization processes, since the 
intersublattice exchange could provide a mechanism for energy transfer from the TM to RE. We consider this in the following section.

\section{E. Effective heating via intersublattice exchange}

Atomistic models have been used previously for the study of short time-scale dynamics ${ }^{43}$ excited by femtosecond laser pulses in ferromagnets, giving good agreement with experimental time scales for the demagnetization process. The model is based on a two-temperature approach ${ }^{44}$ to the calculation of the electron and phonon temperatures $\left(T_{\mathrm{el}}\right.$ and $\left.T_{\mathrm{ph}}\right)$, respectively. The laser power is assumed to be absorbed by the conduction electrons, which are coupled to the phonon system by a phenomenological coupling constant. Because of the difference in heat capacities, this gives a characteristic variation with a rapid initial increase in $T_{\mathrm{el}}$ following the laser pulse, and a slower rise of $T_{\mathrm{ph}}$, with the temperatures reaching equilibrium over time scales on the order of 1-2 ps.

Kazantseva e $_{\text {al }}{ }^{43}$ developed a model of ultrafast heating of a ferromagnet assuming that the energy transfer mechanism is via the conduction electrons into the spin system. However, the recent observations of magnetization reversal in GdFeCo using circularly polarized laser pulses suggests the importance of the development of models of ultrafast reversal in a ferrimagnet. Here the energy transfer channel is less clear-cut. It seems reasonable to assume that there exists a channel via the conduction electrons into the TM spin system as in the pure ferromagnet. However, the mechanism of transfer of energy into the RE sublattice is less clear. Generally the RE provides large anisotropy due to its spin-orbit coupling, however, in the case of bulk Gd there is no spin-orbit coupling of the $4 f$ electrons, however, the $5 d$ electrons have finite spin-orbit coupling partially responsible for the anisotropy. ${ }^{45}$

In this section we investigate the effect of the intersublattice exchange parameter on the demagnetization time in the RE sublattice, which is intrinsically slower due to the higher moment associated with the RE sites, as determined by the magnitude of the correlator. The dynamic model presented here is used in an investigation of the energy transfer induced by the intersublattice exchange.

The approach is to apply a Heaviside step function in temperature from 0 to $300 \mathrm{~K}$ (to which both sublattices are coupled) to simulate the rate of heat transfer to the sublattices. Following the step change we monitor the time evolution of the magnetization changes. The energy transfer is characterized by determining the spin temperature of the RE sublattice, which can be done by mapping the magnetization on to a temperature using the static $M(T)$ curve. We denote this as the effective spin temperature $T_{\text {eff }}$. Figure 10 shows the effective spin temperature of the RE sublattice as a function of time for a range of the intersublattice exchange. As can be seen from Fig. 10, the rate at which the effective spin temperature changes between the low and high exchange values is significantly different, evident from the fact that in the low exchange case in Fig. 10 the RE takes much longer to reach its equilibrium spin temperature. As a result, the intersublattice coupling is seen to be an important energy transfer channel, reducing the longitudinal relaxation of the intrinsically slow relaxing RE impurity.

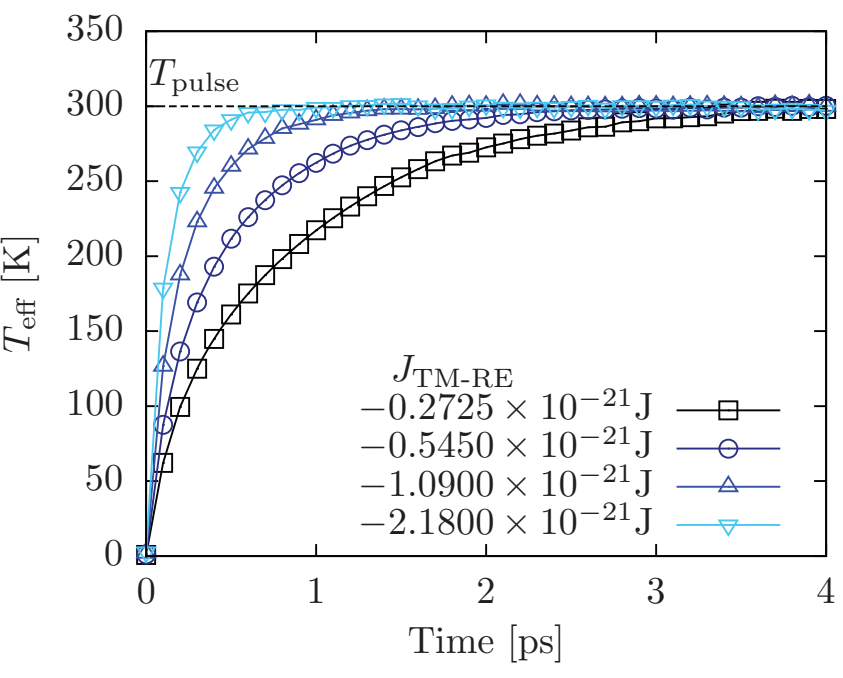

FIG. 10. (Color online) Numerical result of the effective RE spin temperature $\left(T_{\text {eff }}\right)$ using the Langevin dynamics model of the TM-RE ferrimagnet. The system is initially at $0 \mathrm{~K}$, and at time zero a step in temperature to $300 \mathrm{~K}$ is applied. The effective spin temperature is extracted from the equilibrium magnetization. The result shows that the higher exchange leads to an increase in the rate of change of the effective spin temperature in the RE system.

\section{CONCLUSION}

We have developed an atomistic spin model of a TMRE collinear ferrimagnet using a versatile method which is applicable to all alloy types and compositions. The model predictions of static properties, specifically the temperature dependence of the magnetization of each sublattice, were compared with experiments on GdFeCo using the elementspecific XMCD technique. The results of the model are in good agreement with the experimental results and also with the predictions of a mean-field model. In particular, the model predicts some extremely important aspects of this type of ferrimagnet, for example, the magnetization compensation point and the Curie temperature dependence on composition. The role of the compensation point in the effective coercivity was found to be qualitatively similar in the simulations and experiments. We show that the static properties depend on the intersublattice exchange, show its importance for time-resolved dynamics, and show that this interaction may influence laser-induced magnetization dynamics significantly.

Importantly, the model is based on the use of Langevin dynamics, which provides the intrinsic capability to study time-resolved magnetization dynamics in such and other complex systems. The dynamics are potentially important when considering new technologies such as heat-assisted magnetic recording or all-optical magnetization reversal. The TM-RE materials in the amorphous phase, as discussed, are very complex, so more work is required on the details of the crystal structure and the spatial dependence of the exchange constants. Finally, we note that the optomagnetic reversal phenomenon ${ }^{13}$ has only been experimentally demonstrated for amorphous ferrimagnetic alloys. As a result, the development of the current model is potentially important in understanding the fundamental optomagnetic reversal process, and especially the role of the RE component. 
The development of this model will allow us future insights into ultrafast processes in this type of ferrimagnetic material, which we believe to be extremely important for the further development of future technologies.

\section{ACKNOWLEDGMENTS}

The authors are grateful to T. Kachel and C. Stamm (Helmholtz-Zentrum Berlin) for their continuous support with the XMCD measurements. This work has been supported by the EU FP7 programme [Grants No. NMP3-SL-2008214469 (UltraMagnetron), No. 214810 (FANTOMAS), and Grant No. 226716] and Grants No. MAT2007-66719-C0301, FIS2010-20979-C02-02 and No. CS2008-023 from the Spanish Ministry of Science and Education. Financial support from the European COST Action P-19 is also gratefully acknowledged, as well as De Nederlandse Organisatie voor Wetenschappelijk Onderzoek (NWO). *tao500@york.ac.uk

†i.radu@science.ru.nl

${ }^{1}$ M. H. Kryder, E. C. Gage, T. W. McDaniel, W. A. Challener, R. E. Rottmayer, G. Ju, Y.-T. Hsia, and M. F. Erden, Proc. IEEE 96, 1810 (2008).

${ }^{2}$ C. H. Back, W. W. R. Allenspach, S. S. P. Parkin, D. Weller, E. L. Garwin, and H. C. Siegmann, Science 285, 864 (1999).

${ }^{3}$ I. Tudosa, C. Stamm, A. B. Kashuba, F. King, H. C. Siegmann, J. Sthr, G. Ju, B. Lu, and D. Weller, Nature (London) 428, 831 (2004).

${ }^{4}$ J. C. Slonczewski, J. Magn. Magn. Mater. 159, L1 (1996).

${ }^{5}$ T. Devolder, J. Appl. Phys. 98, 053904 (2005).

${ }^{6}$ Y. Acremann, Phys. Rev. Lett. 96, 217202 (2006).

${ }^{7}$ T. Gerrits, H. A. M. van den Berg, J. Hohlfeld, L. Bar, and T. Rasing, Nature (London) 418, 509 (2002).

${ }^{8}$ C. D. Stanciu, F. Hansteen, A. V. Kimel, A. Kirilyuk, A. Tsukamoto, A. Itoh, and Th. Rasing, Phys. Rev. Lett. 99, 047601 (2007).

${ }^{9}$ A. V. Kimel, A. Kirilyuk, A. Tsvetkov, R. V. Pisarev, and Th. Rasing, Nature (London) 429, 850 (2004).

${ }^{10}$ C. D. Stanciu, A. Tsukamoto, A. V. Kimel, F. Hansteen, A. Kirilyuk, A. Itoh, and T. Rasing, Phys. Rev. Lett. 99, 217204 (2007).

${ }^{11}$ A. V. Kimel, A. Kirilyuk, and T. Rasing, Laser Photon. Rev. 1, 275 (2007).

${ }^{12}$ B. Koopmans, M. van Kampen, J. T. Kohlhepp, and W. J. M. de Jonge, Phys. Rev. Lett. 85, 844 (2000).

${ }^{13}$ K. Vahaplar, A. M. Kalashnikova, A. Kimel, D. Hinzke, U. Nowak, R. Chantrell, A. Tsukamoto, A. Itoh, A. Kirilyuk, and T. Rasing, Phys. Rev. Lett. 103, 117201 (2009).

${ }^{14}$ N. Kazantseva, D. Hinzke, U. Nowak, and R. W. Chantrell, Europhys. Lett. 86, 27006 (2009).

${ }^{15}$ M. Mansuripur, The Physical Principles of Magneto-optical Recording (Cambridge University Press, Cambridge, UK, 1995).

${ }^{16}$ P. Hansen, C. Clausen, G. Much, and M. Rosenkranz, J. Appl. Phys. 66, 756 (1989).

${ }^{17}$ L. Néel, Compt. Rend. 203, 304 (1936).

${ }^{18}$ G. M. Buendia and R. Cardona, Phys. Rev. B 59, 6784 (1999).

${ }^{19}$ R. C. Jones, J. Phys. C: Solid State Physics 4, 2903 (1971).

${ }^{20}$ G. A. N. Connell, J. Magn. Magn. Mater. 54, 1561 (1986).

${ }^{21}$ K. H. J. Buschow, Handbook of Magnetic Materials, Vol. 9 (NorthHolland Pub. Co., Amsterdam, 1991).
${ }^{22}$ D. J. Webb, A. F. Marshall, Z. Sun, T. H. Gebelle, and R. M. White, IEEE Trans. Magn. 24, 588 (1988).

${ }^{23}$ R. J. Gambino and J. J. Cuomo, J. Vac. Sci. Technol. 15, 296 (1978).

${ }^{24}$ R. Gambino, N. Saito and Y. Togami, Jpn. J. Appl. Phys. 24, L266 (1985).

${ }^{25}$ Y. Suzuki, S. Takayama, F. Kirino and N. Ohta, IEEE Trans. Magn. 23, 2275 (1987).

${ }^{26}$ W. H. Meiklejohn, Proc. IEEE 74, 1570 (1986).

${ }^{27}$ Y. Suzuki, J. Haimovich and T. Egami, Phys. Rev. B 35, 2162 (1987).

${ }^{28}$ T. L. Gilbert, Phys. Rev. 100, 1243 (1955).

${ }^{29}$ J. L. Garcia-Palacios and F. J. Lázaro, Phys. Rev. B 58, 14937 (1998).

${ }^{30}$ O. Chubykalo-Fesenko, U. Nowak, R. W. Chantrell, and D. Garanin, Phys. Rev. B 74, 094436 (2006).

${ }^{31}$ K. Gilmore, Y. U. Idzerda, and M. D. Stiles, Phys. Rev. Lett. 99, 027204 (2007).

${ }^{32}$ B. Skubic, J. Hellsvik, L. Nordstrom, and O. Eriksson, J. Phys.: Condens. Matter 20, 315203 (2008).

${ }^{33}$ D. Berkov, in The Handbook of Magnetism and Advanced Magnetic Materials, edited by H. Kronmüller and S. Parkin (Wiley, Sussex, 2006).

${ }^{34}$ R. K. Wangsness, Phys. Rev. 91, 1085 (1953).

${ }^{35}$ R. M. Bozorth, Ferromagnetism (IEEE, New York, 2003).

${ }^{36}$ J. Jensen and A. R. Mackintosh, Rare Earth Magnetism: Structures and Excitations (Clarendon, Oxford, 1991).

${ }^{37}$ A. H. Morrish, The Physical Principles of Magnetism (IEEE Press, New York, 2001).

${ }^{38}$ M. Mansuripur, IEEE Trans. Magn 22, 33 (1986).

${ }^{39}$ H. Kachkachi and D. Garanin, Physica A 291, 485 (2001).

${ }^{40}$ T. Kaneyoshi and M. Jascur, J. Photochem. 5, 3254 (1993).

${ }^{41}$ G. S. Rushbrooke, J. G. A. Baker, and P. J. Wood, Phase Transitions and Critical Phenomena (Academic Press, New York, 1974).

${ }^{42}$ N. Kazantseva, D. Hinzke, U. Nowak, R. W. Chantrell, U. Atxitia, and O. Chubykalo-Fesenko, Phys. Rev. B 77, 184428 (2008).

${ }^{43}$ N. Kazantseva, U. Nowak, R. W. Chantrell, J. Hohlfeld, and A. Rebei, Europhys. Lett. 81, 27004 (2008).

${ }^{44}$ J. K. Chen, D. Y. Tzou, and J. E. Beraun, J. Heat Mass Transf. 49, 307 (2006).

${ }^{45}$ M. Colarieti-Tosti, S. I. Simak, R. Ahuja, L. Nordstrom, O. Eriksson, D. Aberg, S. Edvardsson, and M. S. S. Brooks, Phys. Rev. Lett. 91, 157201 (2003). 